\title{
Inhibition of microRNA-155 sensitizes lung cancer cells to irradiation via suppression of HK2-modulated glucose metabolism
}

\author{
XIN LV, LI YAO, JIANLI ZHANG, PING HAN and CUIYUN LI \\ Department of Respiration, Linyi People's Hospital, Linyi, Shandong 276000, P.R. China
}

Received March 11, 2015; Accepted January 11, 2016

DOI: $10.3892 / \mathrm{mmr} .2016 .5394$

\begin{abstract}
MicroRNAs (miRNAs) are small non-coding regulatory RNAs, which are involved in the post-transcriptional regulation of gene expression. miRNA (miR)-155, which has previously been reported to be overexpressed in lung cancer, is correlated with poor patient prognosis. The present study aimed to investigate the effects of miR-155 on the radiosensitivity of human non-small cell lung cancer (NSCLC) cells. To explore the roles of miRNAs in the regulation of irradiation sensitivity of human lung cancer cells, the expressions of miR-155 in response to irradiation, have been studied by RT-qPCR, and the putative direct target of miR-155 was identified by western blot and luciferase assays. The results of the present study revealed that the expression of miR-155 was induced by irradiation, thus suggesting a positive correlation between miR-155 and radiosensitivity. Furthermore, overexpression of miR-155 rendered lung cancer cells resistant to irradiation. In addition, hexokinase 2 (HK2) was identified as an indirect target of miR-155; exogenous overexpression of miR-155 upregulated the expression of HK2, whereas inhibition of miR-155 by antisense miRNA suppressed HK2 expression. In addition, HK2-modulated glucose metabolism was significantly upregulated by overexpression of miR-155. Notably, inhibition of miR-155 sensitized lung cancer cells to irradiation via suppression of glucose metabolism. In conclusion, the present study reported a novel function for miR-155 in the regulation of NSCLC cell radiosensitivity, thus suggesting that miR-155 may be considered a therapeutic target for the development of anticancer drugs.
\end{abstract}

\section{Introduction}

MicroRNAs (miRNAs) are small non-coding regulatory RNAs 17-25 nucleotides long, which are involved in the

Correspondence to: Dr Cuiyun Li, Department of Respiration, Linyi People's Hospital, 1665 Jiefang Road, Lanshan, Linyi, Shandong 276000, P.R. China

E-mail: cuiyunlilinyi@163.com

Key words: microRNA-155, radiosensitivity, non-small cell lung cancer, radioresistance, hexokinase 2 post-transcriptional regulation of gene expression (1). More than $50 \%$ of miRNA genes are located in cancer-associated genomic regions or fragile sites, thus suggesting that miRNAs have important roles in tumor development processes, including apoptosis, invasion, metastasis, proliferation and drug resistance (2). Usually, miRNAs that lead to tumorigenesis are classed as oncomiRs, whereas miRNAs whose functional loss can contribute to the malignant transformation of normal cells, are classed as tumor suppressors (3). miRNA (miR)-155 is one of the most commonly upregulated miRNAs in several types of cancer, including non-small cell lung cancer (NSCLC) (4), breast cancer (5), pancreatic cancer (6), colon cancer (7), renal cancer (8), Hodgkin and B cell lymphoma (9), and secondary acute leukemia (10). Therefore, the dysregulated expression of miR-155 may be an important target for the diagnosis, prognosis and treatment of cancer (11). However, the specific mechanism of action of miR-155 in cancer is currently only partially known.

NSCLC represents the most frequent type of lung cancer, and has a 5-year overall survival rate of $<15 \%$ (12). At present, radiation therapy is regarded as an effective treatment strategy for NSCLC, which uses high-energy rays or particles to destroy lung cancer cells (13). However, radioresistance remains a major barrier that limits the efficacy of radiotherapy (13). Therefore, the development of novel approaches is urgently required, in order to overcome the radioresistance of NSCLC and improve the survival rate of patients. A previous study reported that under hypoxic conditions, miR-155 expression was induced, leading to increased resistance of NSCLC cells to irradiation (14).

The majority of cancer cells exhibit increased glycolysis and dysregulated mitochondrial function, in order to provide sufficient energy for proliferation (15); this unique feature of cancer cells is known as the Warburg effect. Hexokinases (HKs) catalyze the first committed step in glucose metabolism by catalyzing the phosphorylation of glucose to glucose-6-phosphate (G6P). Therefore, hexokinases influence the direction of glucose flux within cells, which is tightly correlated with the processes of tumor initiation and maintenance (15). In addition, elevated glycolysis of cancer cells has been shown to contribute to chemo- and radiotherapy resistance (16). It has previously been reported that radiation induces aerobic glycolysis via reactive oxygen species (17). Furthermore, the oncogene Akt has been shown to promote aerobic glycolysis, which renders cancer cells resistant to irradiation (18), thus 
indicating that glycolysis may be a target for the development of anticancer therapies. The present study investigated the role of miR-155 in the radiosensitivity of NSCLC. The results revealed that inhibition of miR-155 may sensitize NSCLC cells to radiation, thus suggesting that miR-155 may be considered a therapeutic target for the development of anticancer drugs.

\section{Materials and methods}

Cells and cell culture. The A549 and H460 NSCLC cell lines were purchased from American Type Culture Collection (Manassas, VA, USA), and were cultured in Dulbecco's modified Eagle's medium (DMEM) supplemented with $10 \%$ fetal bovine serum, $100 \mathrm{U} / \mathrm{ml}$ penicillin and $100 \mu \mathrm{g} / \mathrm{ml}$ streptomycin (Invitrogen; Thermo Fisher Scientific, Inc., Waltham, MA, USA). All of the cells were cultured at $37^{\circ} \mathrm{C}$ in a humidified incubator containing 95\% air and 5\% $\mathrm{CO}_{2}$, and $\mathrm{A} 549$ and $\mathrm{H} 460$ cells were treated with $3 \mathrm{BrPA}$ at $50 \mu \mathrm{M}$ for $24 \mathrm{~h}$.

Antibody and reagents. The following antibodies were used in the present study: Anti- $\beta$-actin (cat. no. 4967; Cell Signaling Technology, Inc., Danvers, MA, USA) and anti-hexokinase 2 (HK2) (cat. no. 2106; Cell Signaling Technology, Inc.). 3-Bromopyruvate (3BrPA) was purchased from Sigma-Aldrich China, Inc. (Hong-Kong, China). The activities of HK were measured using the Hexokinase Colorimetric Assay kit (Sigma-Aldrich China, Inc.).

Ionizing radiation treatment. Approximately $5 \times 10^{5}$ cells per $6 \mathrm{~cm}$-dish were exposed to various doses of irradiation $(0.2,0.4$, $0.5,0.6,1,1.5,2,2.5,3,4$ and 5 Gys) at room temperature using a Cs-137 irradiator (HWM D-2000; Siemens AG, Munich, Germany) at a dose rate of $2 \mathrm{~Gy} / \mathrm{min}$. The cells were then trypsinized, re-plated in a cell culture dish, and incubated for $16 \mathrm{~h}$, prior to downstream analysis.

Plasmid DNA and miRNA transfections. Transfection was performed using the Lipofectamine 2000 transfection reagent (Invitrogen; Thermo Fisher Scientific, Inc.), according to the manufacturer's protocol. Briefly, $0.5-1 \times 10^{6}$ cells/well were plated onto a 6-well plate, and were incubated overnight until they had reached 70-90\% confluence. The next day, plasmid DNA ( $4 \mu \mathrm{g}$; Addgene, Inc., Cambridge, MA, USA), pre-miR-155 $(100 \mathrm{nM})$ or anti-miR-155 (100 nM) was diluted in Opti-MEM I reduced serum medium (Invitrogen; Thermo Fisher Scientific, Inc.), according to the Lipofectamine 2000 transfection reagent protocol. The cells were then transfected with a HK2 overexpression vector (Addgene, Inc., Cambridge, MA, USA), $100 \mathrm{nM}$ pre-miR-155, anti-miR-155 or negative control using Lipofectamine 2000 and Opti-MEM I reduced serum medium, according to the manufacturer's protocol. The anti-miR negative control used was GMR-miR microRNA inhibitors FAM-labeled single-stranded negative control (Shanghai GenePharma Co., Ltd., Shanghai, China). The precursor and antisense miR-155 were chemically synthesized by Shanghai GenePharma Co., Ltd. (Shanghai, China). A total of $48 \mathrm{~h}$ post transfection, the cells were prepared for further analysis.

$H K$ activity assay. The cells were seeded onto a 6-well plate at a density of $3 \times 10^{5} /$ well overnight. The cells were then collected and $\mathrm{HK}$ activities were measured using the Hexokinase Colorimetric Assay kit (Sigma-Aldrich China, Inc.), according to the manufacturer's protocol.

Glucose consumption and lactate production. The cells were seeded onto a 6-well plate at a density of $3 \times 10^{5} /$ well, and the culture medium was replaced with low glucose DMEM (Invitrogen; Thermo Fisher Scientific, Inc.) after $6 \mathrm{~h}$. The concentrations of glucose and L-lactate were measured after a further $24 \mathrm{~h}$ using a Glucose Test kit (Applygen Technologies, Inc., Beijing, China) and an L-lactate Assay kit (Eton Bioscience, Inc., San Diego, CA, USA), respectively, according to the manufacturer's instructions.

Cell viability assay. A total of $1 \times 10^{4}$ cells/well were seeded onto 48-well plates overnight. The medium was subsequently replaced with fresh medium with or without $3 \mathrm{BrPA}$ at the indicated concentrations, and the cells were incubated for a further $48 \mathrm{~h}$. Cell viability was measured using the MTT assay, according to the manufactory's instructions. Briefly, an equal number of cells were plated into 96 -well plates with culture medium containing either 3BrPA or phosphate-buffered saline (PBS; Sigma-Aldrich China, Inc.) for the untreated control. Following treatments, $0.1 \mathrm{mg} / \mathrm{ml}$ MTT was added to each well and was incubated at $37^{\circ} \mathrm{C}$ for $4 \mathrm{~h}$. Plates were centrifuged at $450 \mathrm{x} \mathrm{g}$ for $5 \mathrm{~min}$ at room temperature, and the medium was discarded. Dimethyl sulfoxide $(0.15 \mathrm{ml}$; Sigma-Aldrich China, Inc.) was added to each well to solubilize the crystals. Absorbance was measured spectrophotometrically at a wavelength of $570 \mathrm{~nm}$ using an ELx800 universal microplate reader (BioTek Instruments, Inc., Winooski, VT, USA).

cDNA preparation and reverse transcription-quantitative polymerase chain reaction $(R T-q P C R)$. Total RNA was extracted following homogenization of the cells using the RNeasy Mini kit (Qiagen Sciences, Inc., Germantown, Maryland MD). DNase digestion was performed during the RNA extraction using the RNase free DNase set (Qiagen, Inc., Valencia, CA, USA). Total RNA ( $1 \mu \mathrm{g})$ was reverse transcribed using the TaqMan microRNA Reverse Transcription kit (Applied Biosystems; Thermo Fisher Scientific, Inc.) and TaqMan microRNA Assays kit (Applied Biosystems; Thermo Fisher Scientific, Inc.), according to the manufacturer's protocols. The precursor miR-155 and RNU6B (served as an internal control) primers used were as follows: miR-155, F 5'-CTAGCC TGCAGGTATTCAAATATTTCCACAGA-3' and R 5'-ATC CGGCCGGCCTGAAGATGGTTATGAACATA-3'; RNU6B, TCGTATCCAGTGCAGGGTCCGAGGTATTCGCACTGGA TACGACAAAATATGGAAC (Invitrogen; Thermo Fisher Scientific, Inc.). The CFX96 Touch Real-Time PCR thermal cycler (Bio-Rad Laboratories, Inc., Hercules, CA, USA) was used with the cycling conditions as follows: $95^{\circ} \mathrm{C}$ for $10 \mathrm{~min}$, $95^{\circ} \mathrm{C}$ for $15 \mathrm{sec}$ and $60^{\circ} \mathrm{C}$ for $60 \mathrm{sec}$, for 40 cycles. All reactions were performed in triplicate. Human U6 served as an internal control. The relative amounts of miRNA were calculated using the comparative cycle quantification method (19).

Western blot analysis. Cells were removed from the treated or non-treated conditions, and were immediately placed on ice. After rinsing with phosphate-buffered saline, the cells were 
A

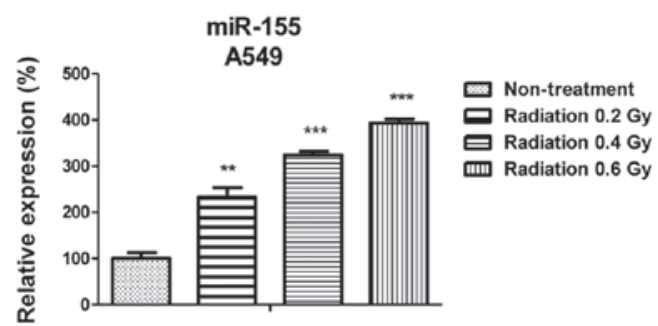

B

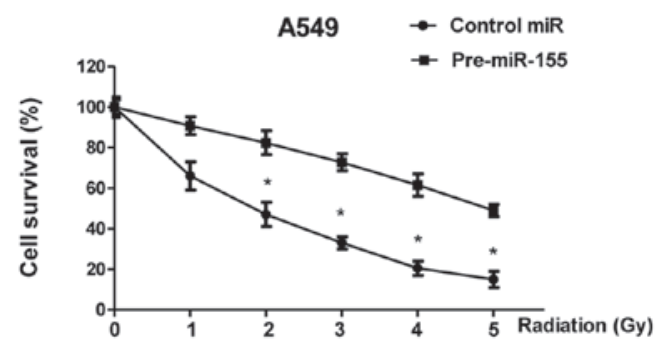

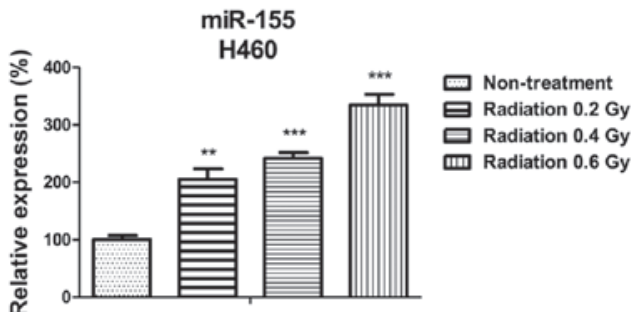

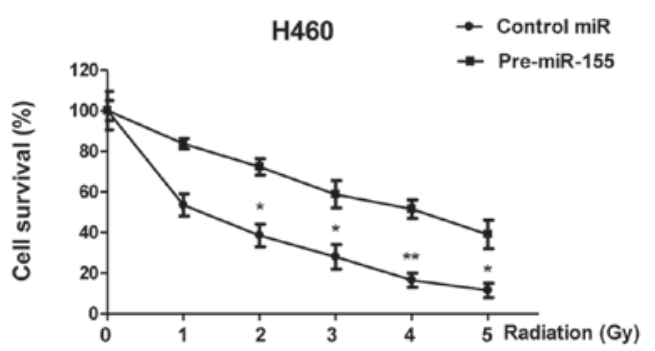

Figure 1. miR-155 is modulated by irradiation in NSCLC cells. (A) A549 (left) and H460 (right) human NSCLC cell lines were treated with irradiation at 0.2 , 0.4 and $0.6 \mathrm{~Gy}$, and the expression levels of miR-155 were measured ${ }^{* *} \mathrm{P}<0.01$ and ${ }^{* * *} \mathrm{P}<0.001$ vs. the non-treatment group. (B) A549 (left) and H460 (right) cells were transiently transfected with pre-miR-155 or control miR for $48 \mathrm{~h}$, and the survival curve was measured upon exposure to the indicated doses of irradiation. ${ }^{*} \mathrm{P}<0.05$ and $^{* *} \mathrm{P}<0.01$ vs. the control miR group. Data are presented as the mean \pm standard error of the mean. miR, microRNA; NSCLC, non-small cell lung cancer.

A

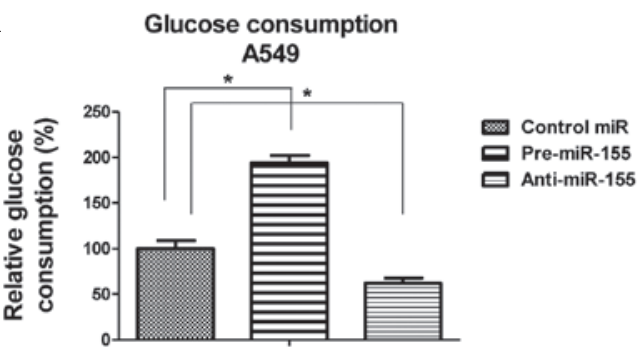

B

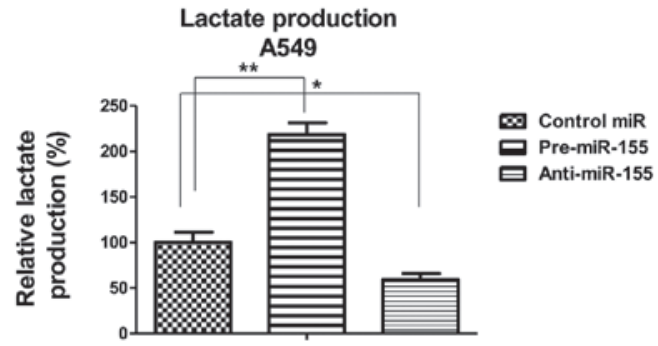

Glucose consumption

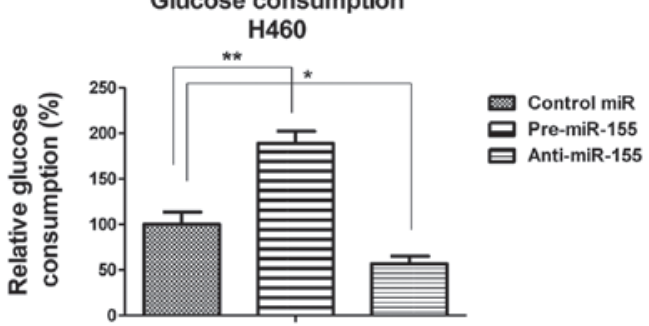

Lactate production

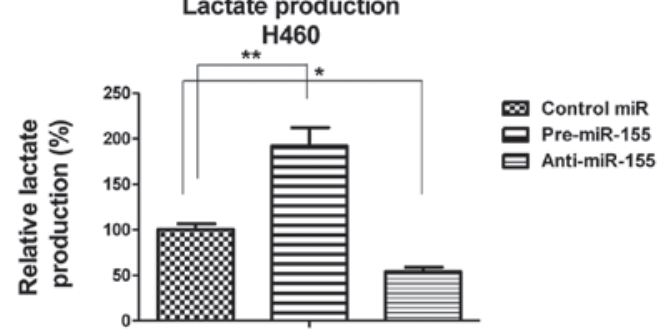

Figure 2. Glucose metabolism of lung cancer cells was upregulated by miR-155. A549 (left) and H460 (right) cells were transfected with pre-miR-155, anti-miR-155 or control miRNA for $48 \mathrm{~h}$ and (A) glucose uptake and (B) lactate production were measured. Data are presented as the mean \pm standard error of the mean. ${ }^{*} \mathrm{P}<0.05 ;{ }^{* *} \mathrm{P}<0.01$. miR, microRNA.

scraped, collected and protein was extracted from them using lysis buffer (Thermo Fisher Scientific, Inc.). Protein concentration was quantified using the Bradford reagent (Sigma-Aldrich China, Inc.) according to the manufacturer's instructions. Total protein $(50 \mu \mathrm{g} / \mathrm{each}$ lane) was loaded and size fractionated by $10 \%$ sodium dodecyl sulfate-polyacrylamide gel electrophoresis, and was transferred to polyvinylidene difluoride membranes (Thermo Fisher Scientific, Inc.). The membranes were incubated with a blocking reagent of 5\% non-fat milk in PBS-Tween 20 (PBST), washed with PBST and probed with primary antibodies $(1: 1,000)$ at $4^{\circ} \mathrm{C}$ overnight. The membranes were then incubated with horseradish peroxidase-conjugated anti-rabbit IgG secondary antibody (1:2,000; cat. no. 7074;
Cell Signaling Technology, Inc.) for $2 \mathrm{~h}$ at room temperature, followed by detection with a Super Signal Enhanced Chemiluminescence kit (Pierce Biotechnology, Inc., Rockford, IL, USA). For sequential blotting, the membranes were stripped with Stripping Buffer (Pierce Biotechnology, Inc.) and re-probed with appropriate antibodies.

Statistical analysis. Statistical evaluation for data analysis was determined by unpaired Student's t-test using the Prism software, version 5.0 (Graphpad Software, Inc., La Jolla, CA, USA). All data are presented as the mean \pm standard error of the mean. $\mathrm{P}<0.05$ was considered to indicate a statistically significant difference. 
A

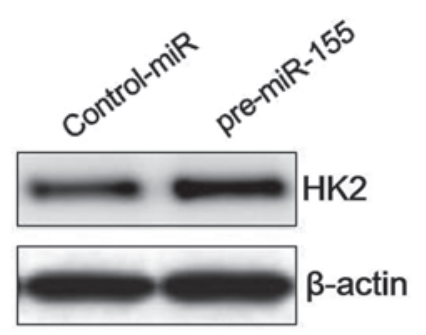

A549

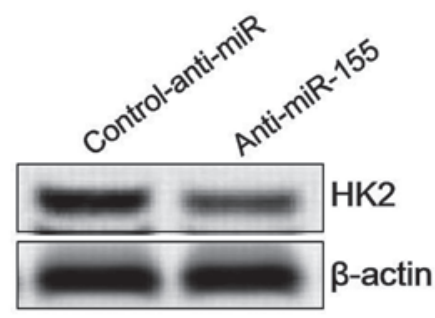

H460
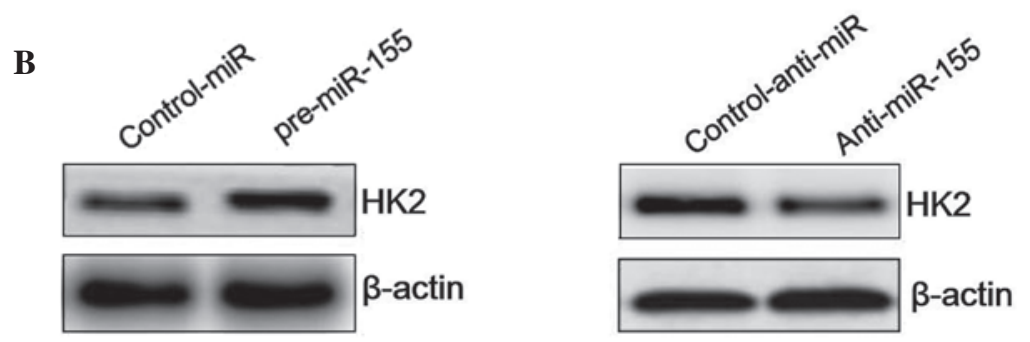

Figure 3. HK2 is upregulated by miR-155. (A) A549 and (B) H460 cells were transfected with pre-miR-155 or control-miR (left), or anti-miR-155 or control-anti-miR (right) for $48 \mathrm{~h}$. The cells were then collected and analyzed by western blotting. $\beta$-actin was used as a loading control. miR, microRNA; HK2, hexokinase 2.

\section{Results}

miR-155 is induced by radiation and correlated with radiation resistance. It has previously been reported that miR-155 levels are increased in numerous types of cancer, including NSCLC, thus suggesting that miR-155 may act as an oncomiR (4). The present study examined the role of miR-155 during the irradiation of cancer cells. Two NSCLC cell lines: A549 and H460, were exposed to numerous non-toxic doses of radiation. Notably, the expression levels of miR-155 were significantly upregulated by irradiation, indicating that miR-155 may have an important role in cancer radiotherapy (Fig 1A). In addition, the present study determined whether miR-155 was able to influence the viability of lung cancer cells in response to radiotherapy. A549 and $\mathrm{H} 460$ cells were transiently transfected with a miR-155 precursor; overexpression of miR-155 in lung cancer cells had a radioprotective effect on A549 and H460 cells (Fig. 1B), as compared with in cells transfected with control miRNA. The half maximal inhibitory concentration of control A549 or H460 cells in response to radiotherapy was $\sim 2 \mathrm{~Gy}$, which was lower than that of the cells overexpressing miR-155 (5 Gy). These results indicate a positive correlation between miR-155 and radioresistance in NSCLC cells.

miR-155 promotes glycolysis of NSCLC cells via the upregulation of $H K 2$. The present study subsequently explored the mechanisms underlying miR-155-mediated radioresistance. As discussed previously, dysregulated or altered energy metabolism is recognized as one of the hallmarks of cancer. Furthermore, it has previously been reported that enhanced aerobic glycolysis of cancer cells contributes to acquired radioresistance $(17,18)$. The present study assessed the rate of glycolysis by measuring glucose consumption and lactate production, which are considered the two major biochemical events of anaerobic glycolysis. As expected, glycolysis was significantly regulated by miR-155:
Overexpression of miR-155 in A549 or H460 cells promoted glucose consumption and lactate production, whereas inhibition of miR-155 by antisense miRNA had the opposite effect (Fig. 2A and B). These results indicate that miR-155-mediated radioresistance may be caused by an upregulation in glycolysis.

miR-155 upregulates the expression of HK2. HK2 is a key enzyme of glycolysis, which catalyzes the formation of glucose-6-phosphate from glucose (20). HK2 activity is positively correlated with the rate of glycolysis. In the present study, HK2 was identified as an indirect target of miR-155 in NSCLC cells (Fig. 3A and B). Overexpression of miR-155 significantly upregulated HK2 protein expression in A549 and H460 cells. Conversely, inhibition of miR-155 downregulated HK2 expression. These results suggest a putative mechanism for the acquired radioresistance of NSCLC cells that overexpress miR-155.

Suppression of glycolysis by HK2 inhibitor sensitizes NSCLC cells to radiation. To investigate whether miR-155-induced glycolysis was due to the upregulation of HK2, lung cancer cells were treated with $3 \mathrm{BrPA}$, which is a specific inhibitor of HK2. Treatment with 3BrPA significantly suppressed the HK activities of A549 and H460 cells (Fig. 4A). Furthermore, the glucose consumption and lactate production of A549 and $\mathrm{H} 460$ cells were decreased following treatment with $3 \mathrm{BrPA}$ $(50 \mu \mathrm{M})$ for $24 \mathrm{~h}$ (Fig. 4B and C). Subsequently, the present study examined whether inhibition of HK2 by $3 \mathrm{BrPA}$ was able to sensitize lung cancer cells to radiation. A549 cells were treated with $3 \mathrm{BrPA}(50 \mu \mathrm{M})$ for $24 \mathrm{~h}$, and were then exposed to various doses of radiation. As expected, A549 cells regained sensitivity to irradiation following treatment with 3BrPA (Fig. 4D). These results suggest that suppression of glycolysis by inhibiting HK2 may be considered a therapeutic strategy to enhance the efficacy of radiotherapy. 
A

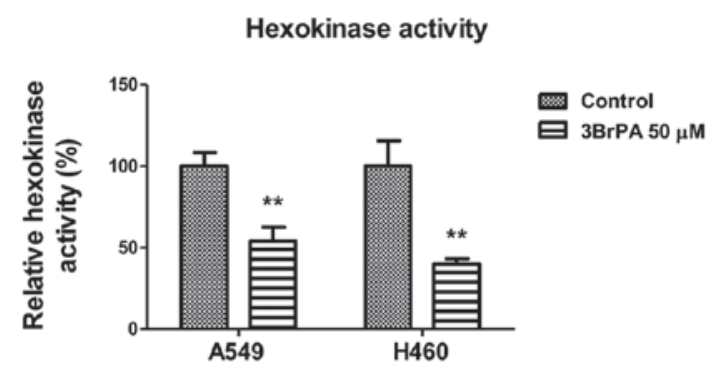

B
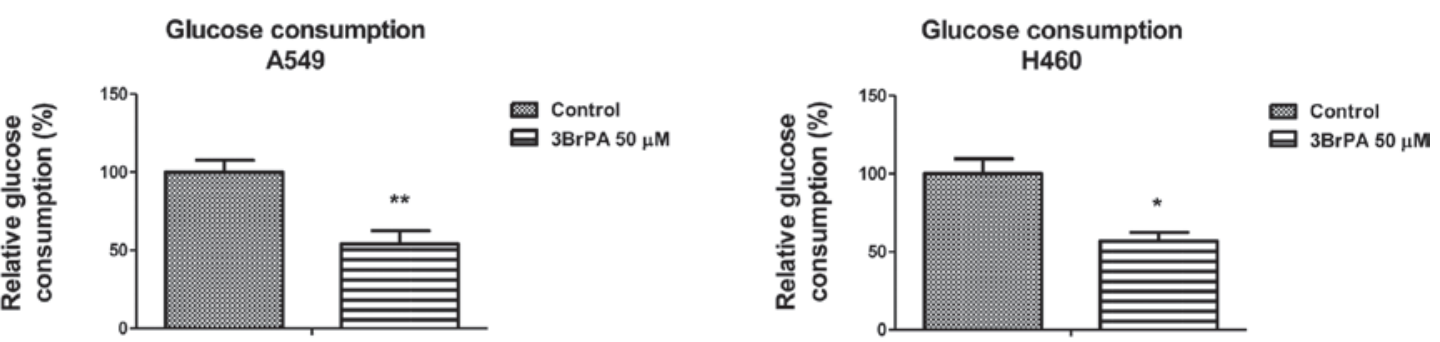

$\mathbf{C}$
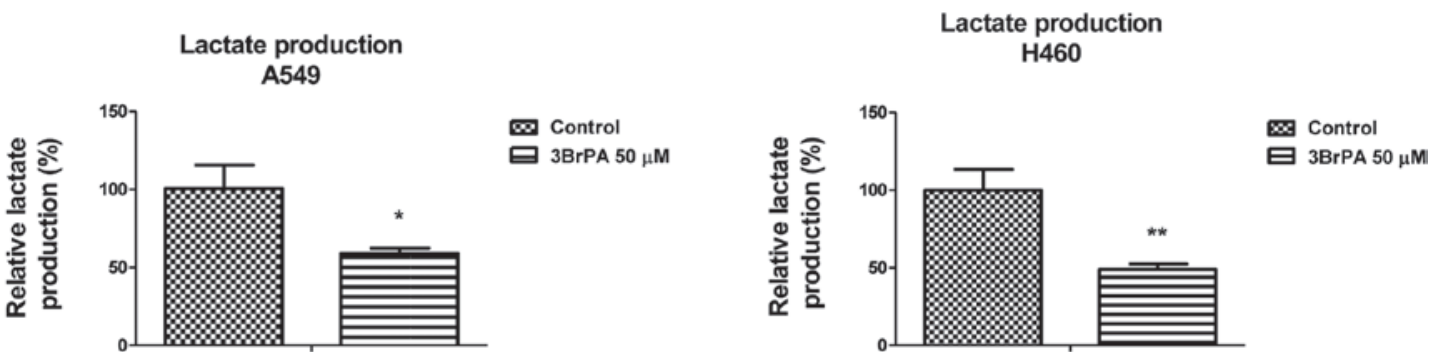

D

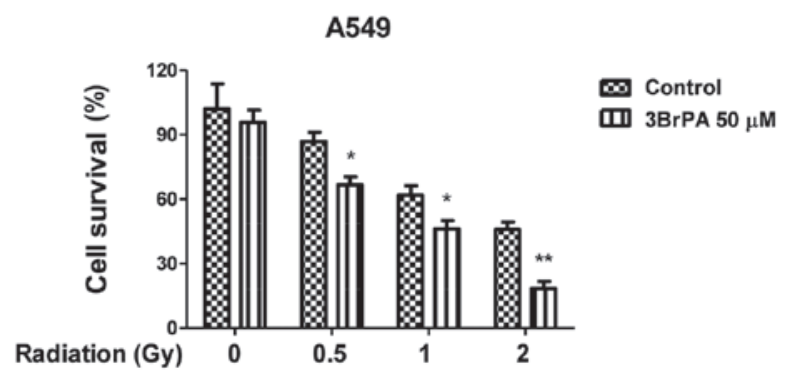

Figure 4. Inhibition of the activity of HK2 sensitizes non-small cell lung cancer cells to irradiation. (A) A549 and $\mathrm{H} 460$ cells were treated with $3 \mathrm{BrPA}(50 \mu \mathrm{M})$ for $24 \mathrm{~h}$. The cells were collected and HK activities were measured. A549 (left) and H460 (right) cells were treated with 3BrPA (50 $\mu \mathrm{M}$ ) for $24 \mathrm{~h}$ and (B) glucose consumption and (C) lactate production were measured. (D) A549 cells were treated with $3 \mathrm{BrPA}(50 \mu \mathrm{M})$ for $24 \mathrm{~h}$, followed by exposure to radiation at $0.5,1$ or 2 Gy. The cells were then analyzed by 3-[4,5 dimethylthiazol-2-yl]-2,5-diphenyltetrazolium bromide assay. Data are presented as the mean \pm standard error of the mean. ${ }^{*} \mathrm{P}<0.05$ and ${ }^{* *} \mathrm{P}<0.01$ vs. the control groups. HK2, hexokinase 2; 3BrPA, 3-bromopyruvate.

Inhibition of miR-155 elevates cancer cell sensitivity to radiation via the suppression of glycolysis. The results of the present study revealed that miR-155 upregulated HK2 expression, which contributed to radioresistance. Subsequently, the radiosensitivity of lung cancer cells was determined in response to various doses of radiation, with or without inhibition of miR-155. As shown in Fig. 5A, inhibition of miR-155 rendered A549 cells more sensitive to irradiation, as compared with the cells transfected with control miRNA. These results are consistent with the previous results that A549 cells are more sensitive to irradiation following treatment with 3BrPA. To determine whether inhibition of miR-155 sensitized lung cancer cells to irradiation via the suppression of glycolysis, anti-miR-155 was transfected into A549 cells undergoing radiation and glucose metabolism was measured. As shown in Fig. 5B, irradiation induced glycolysis, which is consistent with the results of a previous study (17). In addition, under irradiation, inhibition of miR-155 significantly suppressed glucose metabolism (Fig. 5B), thus suggesting that miR-155-mediated radiosensitivity may be associated with the modulation of glycolysis in lung cancer cells.

Restoration of HK2 renders miR-155-inhibited lung cancer cells resistant to irradiation. To further verify the results of the present study, an overexpression vector containing wild type HK2 and antisense miR-155 were co-transfected into A549 cells undergoing radiation, control cells were transfected with a control vector (Fig. 5C). As expected, restoring the expression of HK2 recovered the radioresistance of A549 cells in response to radiation (Fig. 5D). These results clearly 


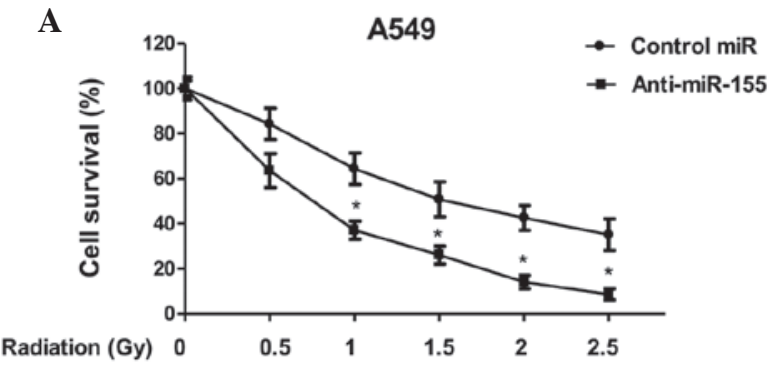

B Glucose consumption
A549

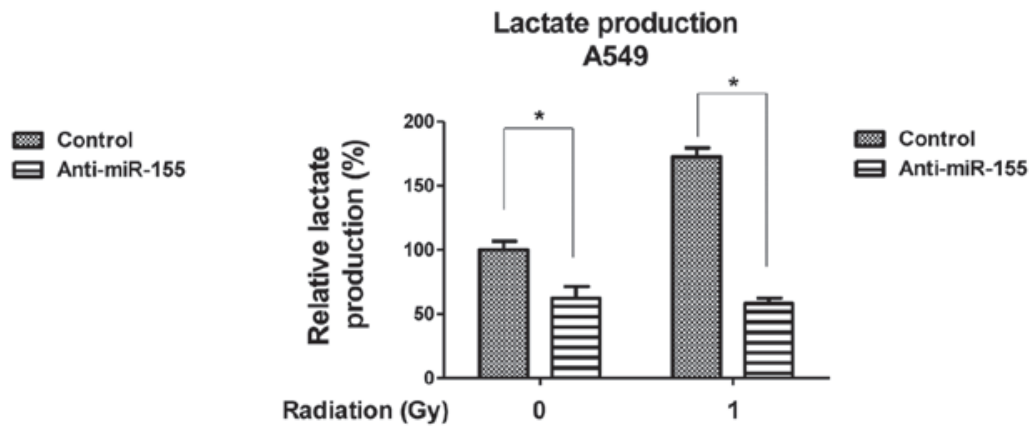

C

D

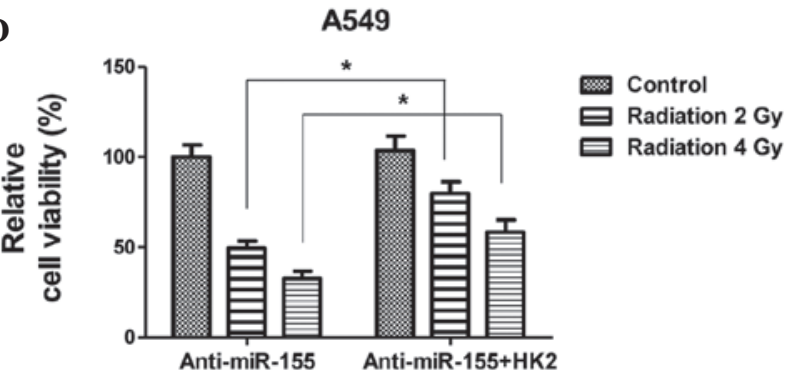

Figure 5. Inhibition of miR-155 sensitizes A549 cells to radiation via the suppression of glycolysis. (A) A549 cells were transfected with anti-miR-155 or control miR for $48 \mathrm{~h}$, followed by exposure to radiation at $0,0.5,1,1.5,2$ or $2.5 \mathrm{~Gy}$. Subsequently, cell survival rates were determined. (B) A549 cells were transfected with anti-miR-155 or control miR for $48 \mathrm{~h}$ followed by exposure to radiation at 0 or $1 \mathrm{~Gy}$. Cells were collected and glucose consumption (left) and lactate production (right) were measured. (C) A549 cells were transfected with control miR, anti-miR-155, or were co-transfected with anti-miR-155 and a HK2 overexpression vector for $48 \mathrm{~h}$. The cells were collected and subjected to western blotting. $\beta$-actin was used as a loading control. (D) A549 cells were transfected with anti-miR-155 alone, or were co-transfected with anti-miR-155 and a HK2 overexpression vector for $48 \mathrm{~h}$, followed by exposure to radiation at 2 and 4 Gy. Subsequently, a 3-[4,5 dimethylthiazol-2-yl]-2,5-diphenyltetrazolium bromide assay was performed. Data are presented as the mean \pm standard error of the mean. ${ }^{\prime} \mathrm{P}<0.05$. miR, microRNA; HK2, hexokinase 2.

indicate that HK2 has an important role in lung cancer cell irradiation sensitivity, and overexpression of miR-155 confers radioresistance at least partially via the indirect upregulation of HK2 expression.

\section{Discussion}

The present study observed that radiation induces the expression of miR-155, which promotes glucose metabolism via the indirect upregulation of HK2. It has previously been reported that miR-155 functions as an important oncomiR, which is a commonly upregulated miRNA in solid and hematological malignancies, including lung cancer, and is correlated with poor patient prognosis (11). The present study demonstrated that miR-155 is induced by radiation, and overexpression of miR-155 in lung cancer cells contributes to radioresistance. In addition, forced inhibition of miR-155 by antisense transfection may render lung cancer cells sensitive to radiation. These results suggested that miR-155 may be correlated with radioresistance, and support the oncogenic roles of miR-155 in NSCLC.

The present study identified an essential role for the miR-155-HK2-glycolysis axis in the Warburg effect. Compared with normal cells, cancer cells exhibit a metabolic switch from oxidative metabolism to anaerobic glycolysis, even in the presence of oxygen. This unique feature of cancer cells is known as the Warburg effect. Activation of the glycolytic pathway is frequently observed in radioresistant cancer cells $(17,18)$. Furthermore, disruption of glycolysis has been considered a possible target for anticancer therapy. It has previously been reported that chemo- and radioresistant cancer cells display an elevated anaerobic glycolysis rate, as compared with parental cells (16), thus indicating that dysregulated glycolysis is correlated with chemo- and radioresistance. At present, whether activation of glycolysis is involved in miRNA regulation remains largely unknown. The present study reported that elevated glycolysis in lung cancer cells by the exogenous expression of miR-155 is correlated with radioresistance. Lung 
cancer cells become increasingly resistant to radiation with increased glycolysis, indicating that dysregulated glycolysis may be a target for overcoming resistance.

Notably, miR-155 was shown to exert its role in glycolysis via upregulation of HK2, which is a key glycolytic enzyme responsible for catalyzing the irreversible first step of glucose metabolism. It has previously been reported that HK2 has an important role in controlling tumorigenesis and chemoresistance (21). Although the indirect regulation of HK2 by the miR-155-miR-143 cluster has been described previously (22), the present study investigated the novel roles of miR-155-mediated glycolysis in radiation resistance via the upregulation of HK2. In our next project, we will focus on the mechanisms underlying radiation-induced miR-155 expression, and screen more differentially regulated miRNAs involved in the radiosensitivity of lung cancer cells. In conclusion, the results of the present study highlight the importance of miR-155 in regulating lung cancer radiosensitivity, and may provide potential targets for the development of cancer therapeutic strategies.

\section{Acknowledgements}

The authors of the present study would like thank the staff and faculty working in the Department of Respiration, Linyi People's Hospital (Linyi, Shandong, China), including Dr Ping Han for providing editorial assistance.

\section{References}

1. Hayes J,Peruzzi PP and LawlerS: MicroRNAs in cancer: Biomarkers, functions and therapy. Trends Mol Med 20: 460-469, 2014.

2. Zheng T, Wang J, Chen X and Liu L: Role of microRNA in anticancer drug resistance. Int J Cancer 126: 2-10, 2010.

3. Ma J, Dong C and Ji C: MicroRNA and drug resistance. Cancer Gene Ther 17: 523-531, 2010.

4. Zang YS, Zhong YF, Fang Z, Li B and An J: MiR-155 inhibits the sensitivity of lung cancer cells to cisplatin via negative regulation of Apaf-1 expression. Cancer Gene Ther 19: 773-778, 2012.

5. Gasparini P, Lovat F, Fassan M, Casadei L, Cascione L, Jacob NK, Carasi S, Palmieri D, Costinean S, Shapiro CL, et al: Protective role of miR-155 in breast cancer through RAD51 targeting impairs homologous recombination after irradiation. Proc Natl Acad Sci USA 111: 4536-4541, 2014

6. Habbe N, Koorstra JB, Mendell JT, Offerhaus GJ, Ryu JK, Feldmann G, Mullendore ME, Goggins MG, Hong SM and Maitra A: MicroRNA miR-155 is a biomarker of early pancreatic neoplasia. Cancer Biol Ther 8: 340-346, 2009.
7. Zhang GJ, Xiao HX, Tian HP, Liu ZL, Xia SS and Zhou T: Upregulation of microRNA-155 promotes the migration and invasion of colorectal cancer cells through the regulation of claudin-1 expression. Int J Mol Med 31: 1375-1380, 2013.

8. Li S, Chen T, Zhong Z, Wang Y, Li Y and Zhao X: microRNA-155 silencing inhibits proliferation and migration and induces apoptosis by upregulating $\mathrm{BACH} 1$ in renal cancer cells. Mol Med Rep 5: 949-954, 2012.

9. Kluiver J, Poppema S, de Jong D, Blokzijl T, Harms G, Jacobs S, Kroesen BJ and van den Berg A: BIC and miR-155 are highly expressed in Hodgkin, primary mediastinal and diffuse large B cell lymphomas. J Pathol 207: 243-249, 2005.

10. Xue H, Hua LM, Guo M and Luo JM: SHIP1 is targeted by miR-155 in acute myeloid leukemia. Oncol Rep 32: 2253-2259, 2014.

11. Czyzyk-Krzeska MF and Zhang X: MiR-155 at the heart of oncogenic pathways. Oncogene 33: 677-678, 2014.

12. Chen Z, Fillmore CM, Hammerman PS, Kim CF and Wong KK: Non-small-cell lung cancers: A heterogeneous set of diseases. Nat Rev Cancer 14: 535-546, 2014.

13. Willers H, Azzoli CG, Santivasi WL and Xia F: Basic mechanisms of therapeutic resistance to radiation and chemotherapy in lung cancer. Cancer J 19: 200-207, 2013.

14. Babar IA, Czochor J, Steinmetz A, Weidhaas JB, Glazer PM and Slack FJ: Inhibition of hypoxia-induced miR-155 radiosensitizes hypoxic lung cancer cells. Cancer Biol Ther 12: 908-914, 2011.

15. Vander Heiden MG, Cantley LC and Thompson CB: Understanding the Warburg effect: The metabolic requirements of cell proliferation. Science 324: 1029-1033, 2009.

16. Zhao Y, Butler EB and Tan M: Targeting cellular metabolism to improve cancer therapeutics. Cell Death Dis 4: e532, 2013.

17. Zhong J, Rajaram N, Brizel DM, Frees AE, Ramanujam N, Batinic-Haberle I and Dewhirst MW: Radiation induces aerobic glycolysis through reactive oxygen species. Radiother Oncol 106: 390-396, 2013.

18. Shimura T, Noma N, Sano Y, Ochiai Y, Oikawa T, Fukumoto M and Kunugita N: AKT-mediated enhanced aerobic glycolysis causes acquired radioresistance by human tumor cells. Radiother Oncol 112: 302-307, 2014.

19. Livak KJ and Schmittgen TD: Analysis of relative gene expression data using real-time quantitative PCR and the 2(-Delta Delta C(T)) Method. Methods 25: 402-408, 2001

20. Mathupala SP, Ko YH and Pedersen PL: Hexokinase-2 bound to mitochondria: Cancer's stygian link to the "Warburg Effect" and a pivotal target for effective therapy. Semin Cancer Biol 19: 17-24, 2009.

21. Suh DH, Kim MA, Kim H, Kim MK, Kim HS, Chung HH, Kim YB and Song YS: Association of overexpression of hexokinase II with chemoresistance in epithelial ovarian cancer. Clin Exp Med 14: 345-353, 2014.

22. Jiang S, Zhang LF, Zhang HW, Hu S, Lu MH, Liang S, Li B, Li Y, Li D, Wang ED and Liu MF: A novel miR-155/miR-143 cascade controls glycolysis by regulating hexokinase 2 in breast cancer cells. EMBO J 31: 1985-1998, 2012. 